\title{
Longitudinal analysis of serum miR-122 in a rat model of Wilson's disease
}

\author{
Ramsi Siaj • Vanessa Sauer · Sandra Stöppeler · Joachim Gerß • \\ Hans-Ullrich Spiegel • Gabriele Köhler • Andree Zibert • \\ Hartmut H.-J. Schmidt
}

Received: 12 October 2011/ Accepted: 25 January 2012/Published online: 11 February 2012

(C) Asian Pacific Association for the Study of the Liver 2012

\begin{abstract}
Purpose MicroRNA-122 (miR-122) has recently been shown to represent a novel biomarker of liver disease. However, the presence of serum miR-122 after liver injury was mostly studied at singular time points. The course of serum miR-122 was determined at consecutive time points during the onset of disease.

Methods Fulminant hepatitis was induced by a highcopper diet in Long-Evans Cinnamon (LEC) rats that were used as models for Wilson's disease (WD). Levels of serum miR-122, alanine aminotransferase (ALT), aspartate aminotransferase (AST), bilirubin, and liver histology were determined.

Results Toxic copper given to isolated hepatocytes induced release of miR-122 into the tissue culture medium. Levels of serum miR-122 were highly elevated $(21.9 \pm 5)$
\end{abstract}

Electronic supplementary material The online version of this article (doi:10.1007/s12072-012-9348-5) contains supplementary material, which is available to authorized users.

R. Siaj · V. Sauer · A. Zibert · H. H.-J. Schmidt ( $\square)$

Klinik und Poliklinik für Transplantationsmedizin,

Universitätsklinikum Münster, Albert-Schweitzer-Campus 1,

Gebäude A14, 48149 Münster, Germany

e-mail: hepar@ukmuenster.de

S. Stöppeler · H.-U. Spiegel

Abteilung Chirurgische Forschung, Klinik und Poliklinik für Allgemein und Viszeralchirurgie, Universitätsklinikum Münster, 48149 Münster, Germany

J. Gerß

Institut für Biometrie und Klinische Forschung,

Universitätsklinikum Münster, 48149 Münster, Germany

G. Köhler

Gerhard-Domagk-Institut für Pathologie, Universitätsklinikum

Münster, 48149 Münster, Germany in LEC rats after high-copper diet in fulminant hepatitis, whereas healthy rats showed low $(<0.6)$ baseline levels of miR-122. Levels of miR-122 in the serum of LEC rats after high-copper diet continuously increased for about 4 weeks prior to the onset of fulminant hepatitis. In most of the animals (77.8\%), significantly increased levels of miR-122 were detected about 2 weeks (13.7 \pm 2 days) earlier as compared to hepatitis-associated serum markers ALT, AST, and bilirubin. Analysis of miR-122 in survivors after cell-based therapy of WD demonstrated a rapid decrease of miR-122 levels following hepatocyte transplantation. miR122 expression in the serum was normalized to baseline levels in most of the (4/5) survivors.

Conclusion Our results suggest that longitudinal analysis of miR-122 allows detection of severe liver disease at an early stage and might be excellently suited to monitor therapy, at least when severe liver disease can be restored as observed after cell-based therapy of WD.

Keywords miR-122 - Biomarker - Fulminant hepatitis . Wilson's disease $\cdot$ Cell-based therapy

\section{Introduction}

MicroRNAs (miRs) are a family of small ( $\sim 22$ nucleotides) non-coding RNAs that regulate post-transcriptional gene expression by targeting messenger RNA transcripts (mRNAs) resulting in cleavage or translational repression $[1,2]$. miRs showed crucial roles in various processes during development, differentiation, and metabolism, and are also involved in diseases like cardiovascular disorders and a variety of cancers [3,4]. miR-122 has been characterized as a liver-specific miR accounting for about $70 \%$ of all cloned miRs of the liver [5]. Interaction of miR-122 
with the $5^{\prime}$-non-coding region of $\mathrm{HCV}$ RNA has been recently related to $\mathrm{HCV}$ infection in vitro and might represent a potential target to inhibit infection [6, 7].

Although the biologic role of miRs is not fully understood, tissue-specific miRs that can be detected in blood upon injury offer the attractive aspect: fluctuation of their serum expression may reflect pathologic transformations of the respective tissue [8]. It was noticed that elevated levels of miR-122 in serum correspond well with a range of liver diseases, including viral infection, fibrosis, and hepatocellular carcinoma (HCC) [3, 9-13]. Serum miR-122 fulfills several criteria for a novel biomarker of liver disease, since it is highly abundant in the liver, almost absent in other organs, released from hepatocytes into the blood upon injury, and sufficiently stable for quantification by common reverse transcription (RT) and real-time PCR methodology. Therefore, it was postulated that miR-122 may represent a highly specific, non-invasive circulating biomarker for liver diseases.

Wilson's disease (WD) is linked to an imbalance of copper homeostasis due to mutations of $A T P 7 B$ copper transporter expressed primarily in the liver [14, 15]. WD is characterized by hepatic insufficiency, acute and chronic hepatitis, and also by various extra-hepatic manifestations [16]. Although being a rare disease, fulminant hepatitis is frequently observed in WD patients and followed by high mortality that accounts for approximately $5 \%$ of acute liver failure (ALF) worldwide. The Long-Evans Cinnamon (LEC) rat harbors a spontaneous mutant of Atp $7 b$ and develops similar symptoms as observed in humans upon dietary copper intake [17-19]. Approximately, 40-60\% of LEC rats develop fulminant hepatitis when fed with commercial chow containing $7-15 \mathrm{mg} / \mathrm{kg}$ of copper [20]. Almost all LEC rats develop fulminant hepatitis when an elevated copper diet is used [21]. The LEC rat is an important model for inherited liver disease to explore novel cell-based therapy of liver, which employs hepatocytes that express functional Atp7b [22, 23]. Following hepatocyte transplantation in LEC rat, WD is almost completely restored due to liver repopulation by $A t p 7 b$ expressing cells. Cell-based therapy was recently introduced to the clinic as a supplemental treatment notably for inherited liver disease [24].

Using samples derived from single time points of liver disease, miR-122 has been characterized as a novel biomarker in animals [9-11] and in patients [3, 12, 13]. We hypothesized that longitudinal analysis of miR-122, during onset of severe liver disease, might allow a further determination of the diagnostic significance of miR-122. The natural sensitivity of LEC rats to develop fulminant hepatitis following dietary copper intake was used to investigate the course of miR-122 expression in the serum of rats. Our findings suggest that miR-122 is best suited to detect early onset of severe liver disease, e.g., fulminant hepatitis, and might be superior to other serum markers used to monitor hepatic disease.

\section{Materials and methods}

Animals and tissue culture

LEC rats that lack functional $A t p 7 b$ and Long-Evans Agouti (LEA) rats expressing wild-type $A t p 7 b$ were a kind gift of S. Gupta (Albert Einstein College of Medicine, New York, USA). Rats were maintained in special animal care facilities at the University Clinic of Münster, Germany, under 12-h light/dark cycle. Rats were genotyped prior to the treatment by PCR [25]. To induce ALF, LEC rats received tap water containing $20 \mathrm{mg} \mathrm{Cu} / 1$ (copper(II)chloride, Sigma-Aldrich) ad libitum and a solid diet (1324, Altromin, Germany) containing $13 \mathrm{mg} \mathrm{Cu} / \mathrm{kg}$. High-copper diet was started at the age of 3 weeks. No detrimental effects of high-copper diet were observed in LEA rats. LEA and LEC rats that received low-copper $(0.3 \mathrm{mg} \mathrm{Cu} / \mathrm{kg})$ solid diet (C1041, Altromin) served as controls. Animals of both groups received distilled tap water ad libitum. Transplantation of hepatocytes into LEC rats was carried out as described in previous works [21]. Briefly, LEC rats that were housed on high-copper diet for 3 weeks received three weekly transplantations of hepatocytes $\left(1 \times 10^{7}\right)$ into the spleen after lateral minilaparotomy. Hepatocytes were derived from LEA rats by standard in situ perfusion technique [26]. Animals were either sham transplanted or received LEA hepatocytes. All protocols for animal use were approved by local authorities.

About $10^{6}$ viable hepatocytes derived by in situ perfusion were incubated in $2 \mathrm{ml}$ of hepatocyte growth medium (Lonza, Germany) for $24 \mathrm{~h}$. Hepatocyte culture was supplemented with copper $(1 \mathrm{mM})$.

\section{miRNA extraction}

Blood sample or medium from isolated rat hepatocytes was centrifuged at $300 \mathrm{~g}$ for $10 \mathrm{~min}$ at $4^{\circ} \mathrm{C}$ to completely remove cellular components, and the supernatant was collected and stored at $-80^{\circ} \mathrm{C}$. RNA was isolated according to a modified protocol of the manufacturer using the miRNasey Mini Kit (Qiagen, Germany). Briefly, RNA was extracted from a $20-\mu$ l supernatant by lysis reagent $(100 \mu \mathrm{l})$ and chloroform $(20 \mu \mathrm{l})$. After centrifugation at $12,000 \mathrm{~g}$ for $15 \mathrm{~min}$ at $4^{\circ} \mathrm{C}$, the aqueous phase was transferred to a fresh tube with $75 \mu$ l of ethanol. Samples were eluted in 50- $\mu$ l RNase-free water and stored at $-80^{\circ} \mathrm{C}$. 


\section{RT and PCR}

RT and quantitative real-time PCR were performed using miScript PCR System (Qiagen, USA) according to the manufacturer's instructions. After purification of RNA in a total volume of $50 \mu \mathrm{l}, 15 \mu \mathrm{l}$ was applied to RT. PCR was carried out in a total volume of $20 \mu \mathrm{l}$ (QuantiTect SYBR Green). PCR was done with miR-122 primer (GenBank ID: NR_031864) or let-7b primer (GenBank ID: NR_031802) in a ABI PRISM ${ }^{\mathrm{TM}}$ 7900HT (PE Applied Biosystems, USA) using the following conditions: $95^{\circ} \mathrm{C}$ for $15 \mathrm{~min}$, $94^{\circ} \mathrm{C}$ for $15 \mathrm{~s}, 55^{\circ} \mathrm{C}$ for $30 \mathrm{~s}$, and $70^{\circ} \mathrm{C}$ for $30 \mathrm{~s}$. Each reaction was performed at least twice. The $C_{\mathrm{t}}$ values of miR-122 expression data were normalized to miR let-7b using the $\Delta C_{\mathrm{t}}$ method. Relative gene expression level normalized to housekeeping gene was determined by equation $2^{\Delta C \mathrm{t}}$, and fold change was calculated.

\section{Serum samples}

Blood was taken retrobulbarly under anesthesia with isoflurane (1-chloro-2, 2, 2,-trifluoroethyl-difluoromethyl ether, Abbott), mixed with oxygen and nitrous oxide. Alanine aminotransferase (ALT) and aspartate aminotransferase (AST) activities and total bilirubin concentration in serum were analyzed photometrically using a Cobas Modular System (Roche Diagnostics, Germany). A modified protocol of Schosinsky et al. [27] was adapted to measure ceruloplasmin oxidase activity in 96-well plates.

\section{Liver histology}

For histologic evaluation, the liver tissues were fixed by immersion for at least 2 days in $4 \%$ formaldehyde solution and were subsequently dehydrated and embedded in paraffin wax to cut serial sections at a thickness of $5 \mu \mathrm{m}$. Polyploidy, steatosis, apoptosis, and proliferation were evaluated by a blinded observer using hematoxylin and eosin stains.

\section{Copper determination}

For determination of liver copper content, samples were dehydrated for $72 \mathrm{~h}$ at $70^{\circ} \mathrm{C}$. Dry weight was determined using analytical balance ME235S (Sartorius, Germany). The liver tissue was incubated in 65\% nitric acid (Merck, Germany), and copper concentration was determined by flame atomic absorption spectroscopy (Shimadzu AA6300, Japan).

\section{Statistical analysis}

Statistical analysis was performed using SPSS 18.0 software. Mean values are presented with standard error (SE).
The statistical analyses were performed by Student's twotailed $t$ test using Bonferroni correction by non-parametric Friedmann test and by receiver operating characteristics (ROC) analysis. A $p$ value $<0.05$ was considered as statistically significant.

\section{Results}

Toxic copper can induce release of miR-122 from hepatocytes

First, we analyzed whether toxic copper leads to release of miR-122 from cultivated hepatocytes. Using tissue culture of primary rat hepatocytes, the presence of miR-122 in the tissue culture medium was determined after addition of toxic copper for $24 \mathrm{~h}$ (Fig. 1). Following copper incubation, most cells were apoptotic and necrotic as reported before [28]. A high concentration of miR-122 was observed in the culture medium as compared to non-treated hepatocytes suggesting that miR-122 was released from dying hepatocytes into the tissue culture medium by toxic copper.

Next, miR-122 expression was determined in serum of animals after high dietary copper intake. LEC rats that are sensitive to copper intake and develop severe hepatic disease were housed on a diet containing high copper [21]. Serum miR-122 was analyzed at different time points of hepatic disease (Table 1). Significantly elevated levels of miR-122 (21.9 \pm 5$)$ were observed in severely diseased $\mathrm{LEC}$ rats $\left(\mathrm{LEC}_{\mathrm{FH}}\right)$ at the time point of fulminant hepatitis (Fig. 2), suggesting that high dietary copper induces release of miR-122 from injured hepatocytes in this rat strain. As in controls, miR-122 expression was determined in LEC rats $\left(\mathrm{LEC}_{\mathrm{L}}\right)$ that received low-copper diet, which was previously shown to prevent hepatic

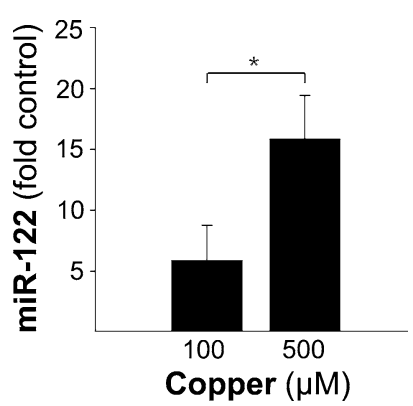

Fig. 1 miR-122 is released from hepatocytes by toxic copper. Hepatocytes $\left(2 \times 10^{5}\right)$ obtained from LEA rats were incubated for $24 \mathrm{~h}$ in cell culture medium containing copper. The factor of miR-122 expression in the medium of copper-treated hepatocytes is given relative to untreated cells $\left(\Delta \Delta C_{\mathrm{t}}\right)$. Mean and standard error is shown $(n=3)$. Asterisks indicate significance $(p<0.05)$. Hepatocytes of LEC rats showed similar levels of miR-122 (data not shown) 
disease [29]. In the $\mathrm{LEC}_{\mathrm{L}}$ rat group, low baseline levels $(0.4 \pm 0.2)$ of miR-122 were found that did not significantly differ from the LEA rat control group $(0.2 \pm 0.2)$, which received high-copper diet. ROC analysis of miR122 in LEC $_{\mathrm{FH}}$ and control groups showed highest AUC value of $1.0(1.0,-1.0)$. A cutoff point set to 11.5 yielded a specificity of $100 \%$ and a sensitivity of $80 \%$. The observation of high miR-122 in LEC $_{\mathrm{FH}}$ compared well with known parameters of WD in LEC rats [20], characterized by increased levels of hepatitis-associated serum markers, low ceruloplasmin activity, and high liver copper, as well as abnormal liver histology (Supplementary Fig. S1).

Serum miR-122 increases significantly earlier as compared to other hepatitis-associated serum markers during the course of WD

After having established that serum miR-122 can be induced in LEC rats by high dietary copper, rats were analyzed on an individual basis from start of high-copper diet up to the time point of fulminant hepatitis when the animals died. Low baseline levels of miR-122 $(<0.6)$ were found up to week 7 (data not shown). Thereafter, individual animals showed continuously increasing levels of miR122. The last three serum samples taken before fulminant hepatitis were inspected for miR-122 expression on an individual basis (Fig. 3). Only 2 out of 18 animals showed miR-122 levels above baseline level at week 8 , whereas all $(18 / 18)$ serum samples showed elevated miR-122 levels at week 12. Notably, at week 10 most of the (77.8\%) animals had significantly elevated miR-122 levels, whereas none of the other hepatitis-associated serum markers (ALT, AST, and bilirubin) reached significance at this early time point suggesting that severe liver disease is detected by miR- 122 2 weeks earlier (13.7 \pm 2 days, median 13.0) than by other markers. ROC analysis of miR-122 at week 10 showed high AUC of $0.9(0.8-1.0)$. A cutoff point set at 0.45

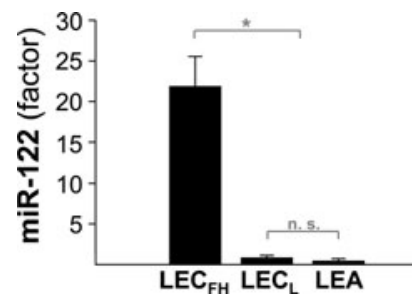

Fig. 2 Serum miR-122 is highly elevated in diseased LEC rats. Serum miR-122 was determined in LEC rats at fulminant hepatitis $\left(\mathrm{LEC}_{\mathrm{FH}}\right)$ and in rats receiving low-copper diet $\left(\mathrm{LEC}_{\mathrm{L}}\right)$. LEA rats that received high copper served as controls. Ten animals were analyzed per group. LEA rats receiving standard chow also showed baseline miR-122 expression (data not shown). Mean and standard error of fold change is shown. Asterisks indicate significance $(p<0.05)$

resulted in a specificity of $85.0 \%$ and a sensitivity of $83.3 \%$ (Supplementary Fig. S2).

miR-122 allows monitoring restoration of disease after cell-based therapy of WD

Transplantation of hepatocytes to LEC rats has been shown to be followed by repopulation of diseased liver and restoration of disease $[22,23]$. We monitored serum levels of miR-122 in LEC rats that survived fulminant hepatitis after having received three hepatocyte transplantations. In the sham-transplanted control group (Fig. 4a), miR-122 levels rapidly increased. Animals died after encountering fulminant hepatitis (week 12). In survivors (Fig. 4b), significantly elevated levels of miR-122 were observed during transplantation (weeks 10-12) that were moderately lower as compared to the sham-transplanted control group (Supplementary Table S1). However, levels of miR-122 declined thereafter in survivors. At week 42, most of the survivors (4/5) showed normalized levels of miR-122. The other hepatitis-associated serum markers also normalized after hepatocyte transplantation in survivors, as previously described for LEC rats $[22,23]$. By this time, a significant restoration of liver copper, ceruloplasmin activity, and

Table 1 Animal groups

\begin{tabular}{llccccc}
\hline Group & Diet $^{\mathbf{a}}$ & Age $^{\mathbf{b}}$ (days) & ALT $(\mathrm{U} / \mathrm{l})$ & AST (U/l) & Bilirubin $(\mathrm{mg} / \mathrm{dl})$ & Animals $(n)$ \\
\hline LEC $_{\mathrm{FH}}$ & High Cu & $84.6 \pm 2$ & $749.4 \pm 113$ & $1132 \pm 124$ & $27.2 \pm 3$ & 10 \\
LEC $_{\mathrm{L}}$ & Low Cu & $98.7 \pm 15$ & $67.2 \pm 5$ & $157.6 \pm 14$ & $0.1 \pm 0$ & 10 \\
LEA & High Cu & $125.1 \pm 1$ & $81.1 \pm 2$ & $145.9 \pm 7$ & $0.1 \pm 0$ & 10 \\
LEC $_{\mathrm{w} 8}^{\mathrm{c}}$ & High Cu & $53.1 \pm 1$ & $100.4 \pm 6$ & $122.4 \pm 3$ & $0.1 \pm 0$ & 18 \\
LEC $_{\mathrm{w} 10}^{\mathrm{c}}$ & High Cu & $68.6 \pm 1$ & $179.6 \pm 43$ & $157.0 \pm 19$ & $0.2 \pm 0$ & 18 \\
LEC $_{\mathrm{w} 12}^{\mathrm{c}}$ & High Cu & $81.7 \pm 2$ & $860.1 \pm 116$ & $1330 \pm 136$ & $28.9 \pm 3$ & 18 \\
\hline
\end{tabular}

Mean \pm SE are given

${ }^{a}$ High- or low-copper diet was started at the age of 3 weeks

b Age at analysis

${ }^{c}$ LEC rats were individually followed up to fulminant hepatitis at week 12 
Fig. 3 Early increase of serum miR-122 during onset of fulminant hepatitis. a Levels of miR-122 are depicted for three consecutive serum samples (weeks 8, 10, and 12).

b-d Levels of hepatitisassociated markers are given. Each dot represents one animal $(n=18)$. Broken lines represent values obtained with healthy LEA rats. Asterisks indicate significance between time points $(p<0.05)$. n.s. not significant
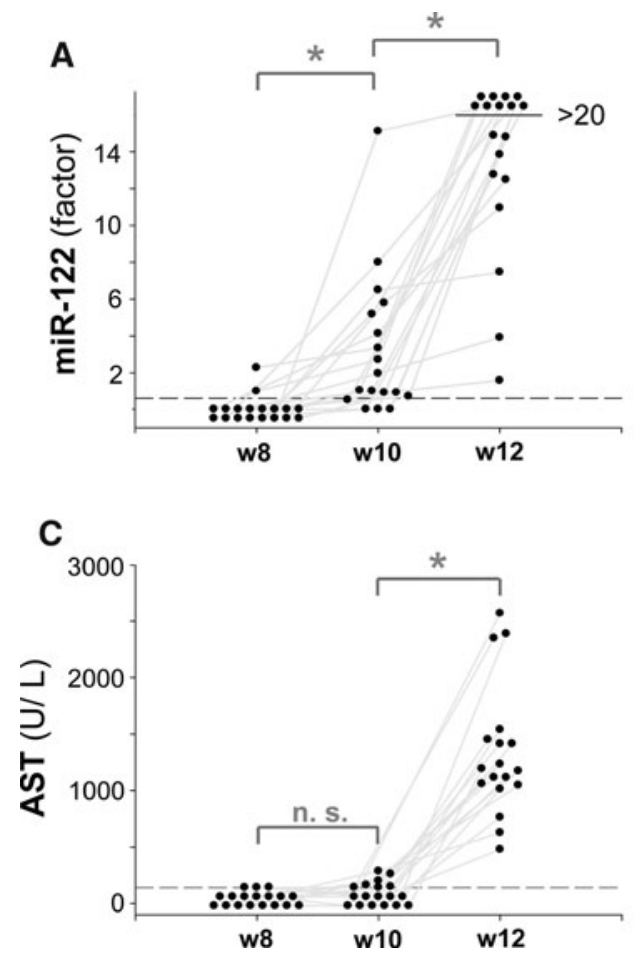
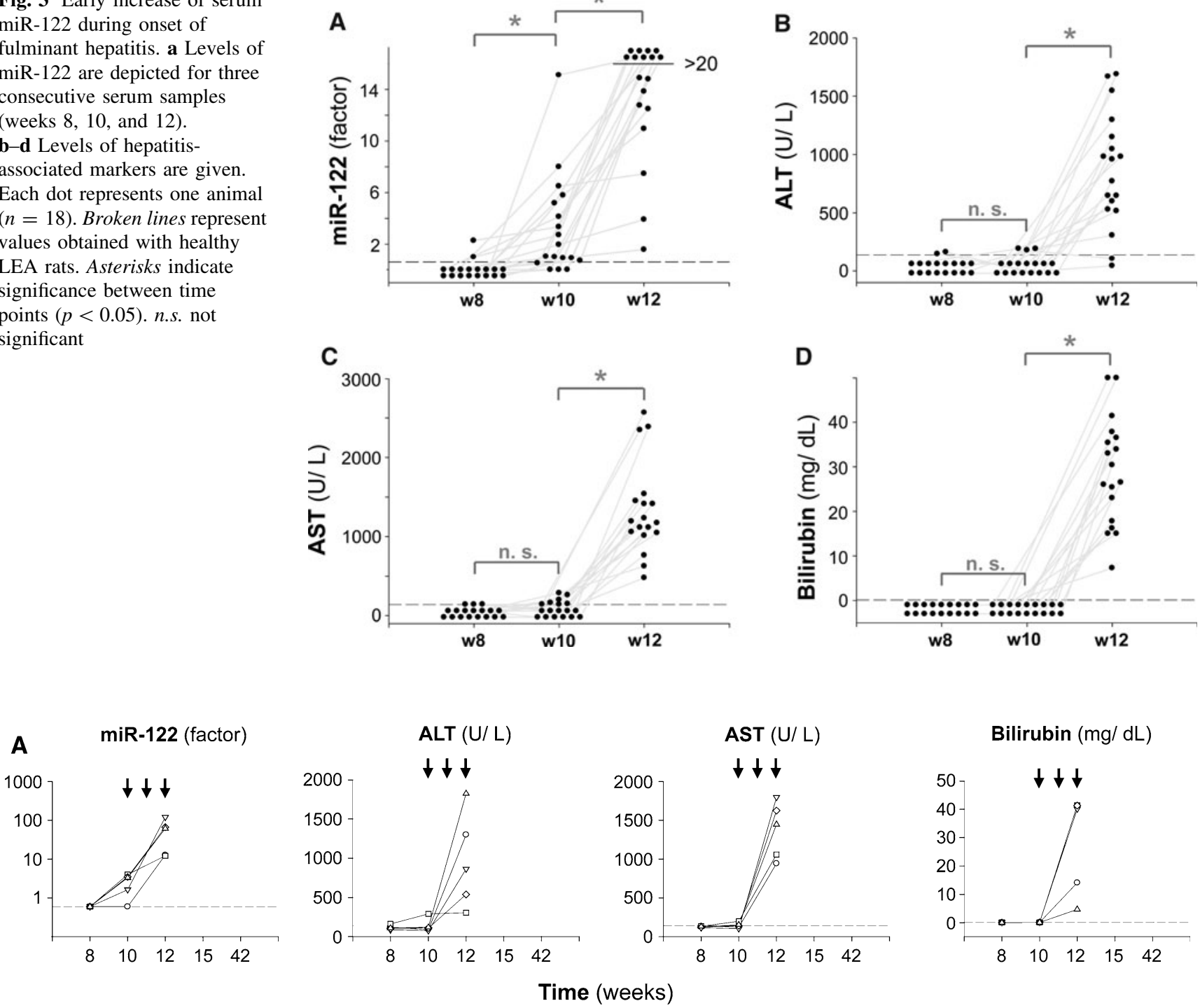

B
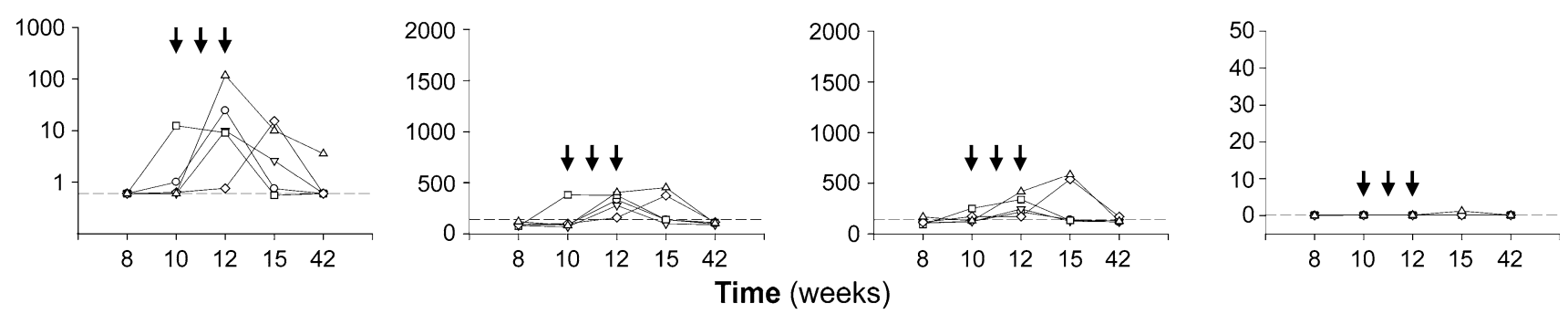

Time (weeks)

Fig. 4 Serum miR-122 allows monitoring of cell-based therapy. Serum miR-122 was determined in LEC rats that were sham transplanted (a) or received hepatocyte transplantation (b). Shamtransplanted animals died at week 12. Arrows indicate the time point of consecutive transplantations that started at week 10. Each line

histology could be observed in the survivors of high-copper diet as shown previously [21]. One survivor showed a moderately increased level $(3.7 \pm 0.1)$ of miR-122 at week 42 , although other hepatitis-associated serum markers normalized. represents one animal. Five animals were monitored per group. The levels of miR-122 and hepatitis-associated markers before week 8 were normal (data not shown). Broken lines represent values obtained with healthy LEA rats

\section{Discussion}

The present study indicates that miR-122 is significantly released from hepatocytes injured by high copper. Detection of serum miR-122 in animals that model WD is 
sensitive and specific. Longitudinal analysis of serum miR122 is highly predictive for course of WD when analyzed on an individual basis. Onset of fulminant hepatitis can be detected almost 2 weeks earlier as compared to established hepatitis-associated serum makers ALT, AST, and bilirubin. Analysis of miR-122 also allows monitoring during therapy of WD and parallels the levels of other hepatitisassociated serum markers. Our observations extend the previous findings of miR-122 in experimental models of liver disease as well as in human disease [9-11, 13], and suggest that longitudinal analyses of serum miR-122 may allow monitoring the course and therapy of severe liver diseases.

miR-122 is highly expressed in hepatocytes, whereas expression is low or absent in other cell types [5, 30]. Our in vitro results using primary rat hepatocytes obtained from LEA rats indicate that miR-122 is released from hepatocytes by toxic copper. Of note, LEA rats are not sensitive to high dietary copper suggesting that elevated (1 mM) copper concentrations as used here or in other in vitro studies can induce cell death in hepatic cells [28, 31]. Copper is proposed to form free radicals that have a high impact on the damage of macromolecules, including proteins and nucleic acids $[32,33]$. As a result, apoptosis and necrosis of tissue culture cells have been reported after toxic copper $[28,34]$, which likely leads to the release of miR-122 into the medium. However, the exact mechanism of copperinduced toxicity of hepatocytes and release of miR-122 has not been understood at the molecular level. As cell death is a common observation following injury, it can be assumed that release of miR-122 by apoptotic and necrotic hepatocytes can be induced by various agents that cause liver disease. Although miR-122 has been recognized as an important regulator of target genes that control cell proliferation, tumorigenesis, and apoptosis [35], the role of miR-122 during the course of WD needs further exploration. In addition, whether hepatocyte-released miR-122 exerts a biological function in liver or other organs remains to be determined. Apart from the exact biologic role of miR-122 in liver, we have shown that release of miR-122 can be used to detect a fulminant course of WD.

The levels of miR-122 were found to be increased in the serum of animals having liver disease following the application of toxins, such as acetaminophen, trichlorobromomethane $\left(\mathrm{CBrCl}_{3}\right)$, carbon tetrachloride $\left(\mathrm{CCL}_{4}\right)$, D-galactosamine hydrochloride (D-GalN) lipopolysaccarides (LPS), and alcohol [9-11]. We investigated whether copper can induce high miR-122 serum levels in the well-characterized rat model of WD where animals naturally develop jaundice, abnormal liver histology, accumulation of liver copper, and ALF even when a normal copper diet that is only moderately lower as in this study [20] is used. A high-copper diet was employed here that induced controlled onset of fulminant hepatitis in all LEC rats around week 12. LEC rats showed high levels of miR-122 at fulminant hepatitis, suggesting that copper-induced toxicity of liver leads to high rates of miR-122 release from injured hepatocytes. Serum miR-122 could be detected by sensitive PCR technique with a high threshold (factor 55-120 as compared to baseline). Notably, asymptomatic LEC rats that were housed on low-copper diet and showed normal liver histology as well as normal levels of serum transaminases [29], displayed slightly increased levels of miR-122, which were, however, not significantly different as compared to healthy LEA rats. Although not investigated here, it is likely that in asymptomatic LEC rats, individual hepatocytes were affected by dietary copper; however, low numbers of affected cells may not allow detection of injury by miR-122 even when highly sensitive PCR analysis is used. Further studies may explore whether there exists a minimal threshold of injured hepatocytes that can be detected by analysis of serum miR-122.

In previous studies, single time points up to 4 days after toxin-induced liver injury were studied in animal models [9-11]. Also, individual time points of well-characterized patients having different stages of chronic $\mathrm{HCV} / \mathrm{HBV}$ infection or non-alcoholic fatty liver disease (NAFLD) $[3,12,13,36,37]$ were investigated. We followed miR-122 levels during the course of WD after dietary copper intake for several weeks. Continuously increasing levels of miR122 were seen in most animals. While low baseline levels of miR-122 were observed up to approximately week 8 in LEC rats, levels of miR-122 rapidly increased, thereafter suggesting that miR-122 was a highly specific marker of severe ongoing liver disease. Significant elevated levels of miR-122 were detected in $\approx 80 \%$ of animals about 2 weeks earlier as compared to the levels of ALT, AST, or bilirubin, suggesting that consecutive follow-up of miR122 may be used to indicate the start of therapy, e.g., early intensification of therapy regimens to prevent severe liver disease. To our knowledge, serum miR-122 levels were analyzed here for the first time in a longitudinal study of liver disease, a strategy that may be applicable to other liver diseases.

WD can be treated by compounds that aim at decoppering, e.g., penicillamine or zinc [16]. Recently, the transplantation of hepatocytes carrying functional Atp $7 b$ has been shown as an alternative to restore disease by repopulation of transplanted hepatocytes in diseased liver [22, 23]. We retrospectively analyzed miR-122 on an individual basis during cell-based therapy of WD in five LEC rats that survived fulminant hepatitis after hepatocyte transplantation [21]. Although significant levels of serum miR-122 were detected before and during the transplantation period suggesting that animals had severe ongoing liver disease, miR-122 levels normalized in most animals 
shortly after transplantation. The course of miR-122 levels was paralleled by an initial increase of serum transaminases in the survivors, followed by normalization of the serum markers suggesting that miR-122 represents an additional biomarker for monitoring of therapy. However, the levels of serum miR-122 may not always correlate to improvement of disease following therapy. In HCV-infected patients, analysis of miR-122 following therapy is complex, e.g., hcv infected due to direct interaction of miR-122 with the genome of HCV and the influence of interferon on the cells [3, 12, 38, 39]. In contrast, a direct correlation of serum miR-122 and ALT could be observed in a portion of $\mathrm{HCV}$-infected patients [37] suggesting that serum miR-122 may represent a valuable biomarker in a subset of patients. Our data suggest that at least for onset of fulminant hepatitis in the course of WD, miR-122 represents a highly specific marker that allows monitoring of therapy. Notably, one survivor showed elevated miR-122 levels at the end of the observation period, although disease was restored in this animal according to the other markers suggesting that miR-122 might have a higher sensitivity for detection of subtle liver injuries. Taken together, our results indicate that follow-up of miR-122 expression in serum on an individual basis may represent a highly specific diagnostic tool, which allows early detection of severe liver disease as well as the monitoring of disease restoration after therapy of inherited liver disease.

Acknowledgements RS and VS were supported partly by the "Deutsche Forschungsgemeinschaft" (DFG; grant SCHM 964/10-1) and by the "Innovative Medizinische Forschung" Münster (IMF). We thank G. Chandhok for critically reading the manuscript and K. Cebulla for technical assistance.

\section{References}

1. Bartel DP. MicroRNAs: target recognition and regulatory functions. Cell 2009;136:215-233

2. Krol J, Loedige I, Filipowicz W. The widespread regulation of microRNA biogenesis, function and decay. Nat Rev Genet 2010;11:597-610

3. Morita K, Taketomi A, Shirabe K, et al. Clinical significance and potential of hepatic microRNA-122 expression in hepatitis $\mathrm{C}$. Liver Int 2011;31:474-484

4. Sayed D, Abdellatif M. MicroRNAs in development and disease. Physiol Rev 2011;91:827-887

5. Allen KJ, Cheah DM, Wright PF, et al. Liver cell transplantation leads to repopulation and functional correction in a mouse model of Wilson's disease. J Gastroenterol Hepatol 2004;19:1283-1290

6. Jopling CL, Yi M, Lancaster AM, Lemon SM, Sarnow P. Modulation of hepatitis $\mathrm{C}$ virus RNA abundance by a liver-specific MicroRNA. Science 2005;309:1577-1581

7. Lanford RE, Hildebrandt-Eriksen ES, Petri A, et al. Therapeutic silencing of microRNA-122 in primates with chronic hepatitis C virus infection. Science 2010;327:198-201
8. Mitchell PS, Parkin RK, Kroh EM, et al. Circulating microRNAs as stable blood-based markers for cancer detection. Proc Natl Acad Sci USA 2008;105:10513-10518

9. Wang K, Zhang S, Marzolf B, et al. Circulating microRNAs, potential biomarkers for drug-induced liver injury. Proc Natl Acad Sci USA 2009; 106:4402-4407

10. Laterza OF, Lim L, Garrett-Engele PW, et al. Plasma MicroRNAs as sensitive and specific biomarkers of tissue injury. Clin Chem 2009;55:1977-1983

11. Zhang Y, Jia Y, Zheng R, et al. Plasma microRNA-122 as a biomarker for viral-, alcohol-, and chemical-related hepatic diseases. Clin Chem 2010;56:1830-1838

12. Marquez RT, Bandyopadhyay S, Wendlandt EB, et al. Correlation between microRNA expression levels and clinical parameters associated with chronic hepatitis $\mathrm{C}$ viral infection in humans. Lab Invest 2010;90:1727-1736

13. Xu J, Wu C, Che X, et al. Circulating microRNAs, miR-21, miR122 , and miR-223, in patients with hepatocellular carcinoma or chronic hepatitis. Mol Carcinog 2011;50:136-142

14. Tanzi RE, Petrukhin K, Chernov I, et al. The Wilson disease gene is a copper transporting ATPase with homology to the Menkes disease gene. Nat Genet 1993;5:344-350

15. Bull PC, Thomas GR, Rommens JM, Forbes JR, Cox DW. The Wilson disease gene is a putative copper transporting P-type ATPase similar to the Menkes gene. Nat Genet 1993;5:327-337

16. Ala A, Walker AP, Ashkan K, Dooley JS, Schilsky ML. Wilson's disease. Lancet 2007;2007(369):397-408

17. Yoshida MC, Masuda R, Sasaki M, et al. New mutation causing hereditary hepatitis in the laboratory rat. J Hered 1987;78: 361-365

18. Wu J, Forbes JR, Chen HS, Cox DW. The LEC rat has a deletion in the copper transporting ATPase gene homologous to the Wilson disease gene. Nat Genet 1994;7:541-545

19. Sasaki N, Hayashizaki Y, Muramatsu M, et al. The gene responsible for LEC hepatitis, located on rat chromosome 16, is the homolog to the human Wilson disease gene. Biochem Biophys Res Commun 1994;202:512-518

20. Li Y, Togashi Y, Sato S, et al. Spontaneous hepatic copper accumulation in Long-Evans Cinnamon rats with hereditary hepatitis. A model of Wilson's disease. J Clin Invest 1991;87: 1858-1861

21. Sauer V, Siaj R, Stöppeler S, et al. Repeated transplantation of hepatocytes prevents fulminant hepatitis in a rat model of Wilson's disease. Liver Transpl (in press)

22. Yoshida Y, Tokusashi Y, Lee GH, Ogawa K. Intrahepatic transplantation of normal hepatocytes prevents Wilson's disease in Long-Evans cinnamon rats. Gastroenterology 1996;111:16541660

23. Irani AN, Malhi H, Slehria S, et al. Correction of liver disease following transplantation of normal rat hepatocytes into LongEvans Cinnamon rats modeling Wilson's disease. Mol Ther 2001;3:302-309

24. Dhawan A, Strom SC, Sokal E, Fox IJ. Human hepatocyte transplantation. Methods Mol Biol 2010;640:525-534

25. Ahmed S, Deng J, Borjigin J. A new strain of rat for functional analysis of PINA. Brain Res Mol Brain Res 2005;137:63-69

26. Berry MN, Friend DS. High-yield preparation of isolated rat liver parenchymal cells: a biochemical and fine structural study. J Cell Biol 1969;43:506-520

27. Schosinsky KH, Lehmann HP, Beeler MF. Measurement of ceruloplasmin from its oxidase activity in serum by use of $o$-dianisidine dihydrochloride. Clin Chem 1974;20:1556-1563

28. Sauer V, Siaj R, Todorov T, Zibert A, Schmidt HH. Overexpressed ATP7B protects mesenchymal stem cells from toxic copper. Biochem Biophys Res Commun 2010;395:307-311 
29. Sugawara N, Sugawara C. A copper deficient diet prevents hepatic copper accumulation and dysfunction in Long-Evans Cinnamon (LEC) rats with an abnormal copper metabolism and hereditary hepatitis. Arch Toxicol 1994;69:137-140

30. Chang J, Nicolas E, Marks D, et al. miR-122, a mammalian liverspecific microRNA, is processed from hor mRNA and may downregulate the high affinity cationic amino acid transporter CAT-1. RNA Biol 2004;1:106-113

31. Schilsky ML, Blank RR, Czaja MJ, et al. Hepatocellular copper toxicity and its attenuation by zinc. J Clin Invest 1989;84: 1562-1568

32. Britton RS. Metal-induced hepatotoxicity. Semin Liver Dis 1996;16:3-12

33. Camakaris J, Voskoboinik I, Mercer JF. Molecular mechanisms of copper homeostasis. Biochem Biophys Res Commun 1999;261: 225-232

34. Strand S, Hofmann WJ, Grambihler A, et al. Hepatic failure and liver cell damage in acute Wilson's disease involve CD95 (APO1/Fas) mediated apoptosis. Nat Med 1998;4:588-593
35. Huang $\mathrm{S}, \mathrm{He} \mathrm{X}$. The role of microRNAs in liver cancer progression. Br J Cancer 2011;104:235-340

36. Cermelli S, Ruggieri A, Marrero JA, Ioannou GN, Beretta L. Circulating MicroRNAs in patients with chronic hepatitis $\mathrm{C}$ and non-alcoholic fatty liver disease. PLoS One 2011;6:e23937

37. Bihrer V, Friedrich-Rust M, Kronenberger B, et al. Serum miR-122 as a biomarker of necroinflammation in patients with chronic hepatitis C virus infection. Am J Gastroenterol 2011;106:1663-1669

38. Sarasin-Filipowicz M, Krol J, Markiewicz I, Heim MH, Filipowicz W. Decreased levels of microRNA miR-122 in individuals with hepatitis $C$ responding poorly to interferon therapy. Nat Med 2009;15:31-33

39. Pedersen IM, Cheng G, Wieland S, et al. Interferon modulation of cellular microRNAs as an antiviral mechanism. Nature 2007;449: 919-922 